\title{
Implicit Numerical Solutions for Solving Stochastic Differential Equations with Jumps
}

\author{
Ying Du and Changlin Mei \\ School of Mathematics and Statistics, Xian Jiaotong University, Xian, Shaanxi 710049, China \\ Correspondence should be addressed to Ying Du; duyingxjtu@aliyun.com
}

Received 25 January 2014; Accepted 26 June 2014; Published 24 July 2014

Academic Editor: Maoan Han

Copyright (C) 2014 Y. Du and C. Mei. This is an open access article distributed under the Creative Commons Attribution License, which permits unrestricted use, distribution, and reproduction in any medium, provided the original work is properly cited.

\begin{abstract}
To realize the applications of stochastic differential equations with jumps, much attention has recently been paid to the construction of efficient numerical solutions of the equations. Considering the fact that the use of the explicit methods often results in instability and inaccurate approximations in solving stochastic differential equations, we propose two implicit methods, the $\theta$-Taylor method and the balanced $\theta$-Taylor method, for numerically solving the stochastic differential equation with jumps and prove that the numerical solutions are convergent with strong order 1.0. For a linear scalar test equation, the mean-square stability regions of the methods are derived. Finally, numerical examples are given to evaluate the performance of the methods.
\end{abstract}

\section{Introduction}

Stochastic differential equations (SDEs) have been one of the most important mathematical tools for dealing with many problems in a variety of practical areas. However, SDEs are in general so complex that the analytical solutions can rarely be obtained. Thus, it is a common way to numerically solve SDEs. Since the explicit numerical methods often result in instability and inaccurate approximations to the solutions unless the step-size is very small, it is often necessary to use some implicit methods in numerically solving SDEs.

Generally speaking, there are two kinds of implicit numerical methods. One is the semi-implicit methods in which the drift components are computed implicitly while the diffusion components are computed explicitly. Higham $[1,2]$ studied the stochastic $\theta$-method for SDEs and SDEs with jumps (SDEJs). When $\theta=1$, the stochastic $\theta$-method is the backward Euler method. The backward Euler method is discussed in [3-5] and the references therein. Hu and Gan [6] proposed a class of drift-implicit one-step methods for neutral stochastic delay differential equations with jump diffusion. Higham and Kloeden [3, 7] constructed the splitstep backward Euler method and the compensated splitstep backward Euler method for SDEJs. Ding et al. [8] introduced the split-step $\theta$-method which is more general than the split-step backward Euler method. Wang and Gan [9] studied split-step one-leg $\theta$ methods for SDEs. Buckwar and Sickenberger [10] compared the mean-square stability properties of the $\theta$-Maruyama and $\theta$-Milstein methods for SDEs.

The other is the fully implicit methods in which both the drift components and the diffusion components are computed implicitly. Since implicit stochastic terms in the implicit methods lead to infinite absolute moments of the numerical solution, extensive research has been done to address this issue [11-26]. For example, Milstein et al. [11] proposed the balanced implicit method for the numerical solutions of SDEs. Burrage and Tian [12] suggested three implicit Taylor methods: the implicit Euler-Taylor method with strong order 0.5 , the implicit Milstein-Taylor method with strong order 1.0, and the implicit Taylor method with strong order 1.5. Kahl and Schurz [16] introduced the balanced Milstein method for ordinary SDEs. Wang and Liu $[20,21]$ proposed the semi-implicit Milstein method and the split-step backward balanced Milstein method for stiff stochastic systems. Furthermore, Haghighi and Hosseini [23] developed a class of general split-step balanced numerical methods for SDEs. 
Let $\left(\Omega, \mathscr{F},\left\{\mathscr{F}_{t}\right\}_{t \in\left[t_{0}, T\right]}, \mathbb{P}\right)$ be a complete probability space with the filtration $\left\{\mathscr{F}_{t}\right\}_{t \in\left[t_{0}, T\right]}$ satisfying the usual conditions that $\mathscr{F}_{t}$ is right-continuous and $\mathscr{F}_{0}$ contains all $\mathbb{P}$-null sets. In this paper, we consider the stochastic differential equations with jumps of the form

$$
\begin{array}{r}
\mathrm{d} x(t)=f(x(t)) \mathrm{d} t+g(x(t)) \mathrm{d} W(t)+h(x(t)) \mathrm{d} N(t), \\
t \in\left[t_{0}, T\right] ; \\
x\left(t_{0}\right)=x_{0},
\end{array}
$$

where $W(t)$ is $\mathscr{F}_{t}$-adapted Wiener process and $N(t)$ is a scalar poisson process with intensity $\lambda$ and is independent of $W(t)$. $\mathrm{Hu}$ and Gan [22, 25] proposed the balanced method for SDEJs (1) and stochastic pantograph equations with jumps, respectively, and proved that the numerical solution converges to the analytical solution with rate $1 / 2$. The asymptotic stability of the balanced method for SDEJs (1) was obtained in [26]. To obtain higher order numerical schemes and improve the accuracy of the numerical solutions, we propose two kinds of implicit Taylor methods and prove that the numerical solutions converge to the true solutions of SDEJs (1) with rate 1.0 .

The rest of the paper is arranged as follows. In Section 2, we introduce the $\theta$-Taylor methods and the fully implicit balanced $\theta$-Taylor methods for SDEJs (1). The strong convergence properties of these implicit methods are proved in Section 3. The mean-square stability of the numerical solutions is discussed in Section 4. Some numerical experiments are performed in Section 5 to evaluate the performance of the proposed numerical methods.

\section{The Numerical Methods}

Define a mesh $0 \leq t_{0}<t_{1}<\cdots<t_{n}<t_{n+1}<\cdots<t_{N}=T$ on the time interval $\left[t_{0}, T\right]$ with $t_{n}=n \Delta$ and the step-size $\Delta=T / N \cdot x_{n}$ is the numerical approximation to $x\left(t_{n}\right)$. Based on appropriate stochastic Taylor expansions, Maghsoodi [27] generalized the Milstein scheme to SDEJs and obtained the order 1.0 strong Taylor scheme (Taylor for short) as

$$
\begin{aligned}
x_{n+1}= & x_{n}+f\left(x_{n}\right) \Delta+g\left(x_{n}\right) \Delta W_{n} \\
& +\frac{1}{2} g\left(x_{n}\right) g^{\prime}\left(x_{n}\right)\left[\left(\Delta W_{n}\right)^{2}-\Delta\right] \\
& +\frac{1}{2}\left(3 h\left(x_{n}\right)-h_{h}\left(x_{n}\right)\right) \Delta N_{n} \\
& +\left(g_{h}\left(x_{n}\right)-g\left(x_{n}\right)\right) \Delta W_{n} \Delta N_{n} \\
& +\frac{1}{2}\left(h_{h}\left(x_{n}\right)-h\left(x_{n}\right)\right)\left(\Delta N_{n}\right)^{2} \\
& +\left(g\left(x_{n}\right) h^{\prime}\left(x_{n}\right)-g_{h}\left(x_{n}\right)+g\left(x_{n}\right)\right) \Delta Z_{n},
\end{aligned}
$$

where $g_{h}(x)=g(x+h(x)), h_{h}(x)=h(x+h(x))$, and $\Delta Z_{n}=$ $\int_{t_{n}}^{t_{n+1}} \int_{t_{n}}^{s} \mathrm{~d} W_{t} \mathrm{~d} N_{s}=\int_{t_{n}}^{t_{n+1}}\left(W_{s}-W_{t_{n}}\right) \mathrm{d} N_{s}$.
Note that $\Delta Z_{n}=\sum_{j}\left(W\left(\tau_{j}\right)-W\left(t_{k}\right)\right)=\sum_{j=N_{n}+1}^{N_{n+1}}\left(N_{n+1}-j+\right.$ 1) $\left(W\left(\tau_{j}\right)-W\left(\tau_{j-1}\right)\right)$ [28]. Given a jump time $\tau_{j}$ in $\left[t_{n}, t_{n+1}\right)$, $\Delta Z_{1 n}\left(\tau_{j}\right)=W\left(\tau_{j}\right)-W\left(\tau_{j-1}\right) \sim N\left(0, \tau_{j}-\tau_{j-1}\right)\left(N_{n}+1 \leq j \leq\right.$ $\left.N_{n+1}\right)$. In addition, the random variable $\Delta W_{n}=W\left(t_{n+1}\right)-$ $W\left(t_{n}\right)$ is dependent on $\Delta Z_{1 n}\left(\tau_{j}\right)$, and its sample values can be calculated by $\Delta W_{n}=\sum_{j=N_{n}+1}^{N_{n+1}} \Delta Z_{1 n}\left(\tau_{j}\right)+\Delta Z_{1 n}\left(t_{n+1}\right)$ where $\Delta Z_{1 n}\left(t_{n+1}\right)=W\left(t_{n+1}\right)-W\left(\tau_{N_{n+1}}\right) \sim N\left(0, t_{n+1}-\tau_{N_{n+1}}\right)$.

By changing the explicit deterministic term into implicit term, we have the following $\theta$-Taylor method:

$$
\begin{aligned}
x_{n+1}= & x_{n}+\Delta\left[(1-\theta) f\left(x_{n}\right)+\theta f\left(x_{n+1}\right)\right] \\
& +g\left(x_{n}\right) \Delta W_{n}+\frac{1}{2} g\left(x_{n}\right) g^{\prime}\left(x_{n}\right)\left[\left(\Delta W_{n}\right)^{2}-\Delta\right] \\
& +\frac{1}{2}\left(3 h\left(x_{n}\right)-h_{h}\left(x_{n}\right)\right) \Delta N_{n} \\
& +\left(g_{h}\left(x_{n}\right)-g\left(x_{n}\right)\right) \Delta W_{n} \Delta N_{n} \\
& +\frac{1}{2}\left(h_{h}\left(x_{n}\right)-h\left(x_{n}\right)\right)\left(\Delta N_{n}\right)^{2} \\
& +\left(g\left(x_{n}\right) h^{\prime}\left(x_{n}\right)-g_{h}\left(x_{n}\right)+g\left(x_{n}\right)\right) \Delta Z_{n} .
\end{aligned}
$$

Note that the $\theta$-Taylor method (3) becomes the Taylor method (2) when $\theta=0$.

Using the idea of the balanced implicit method and combining it with the $\theta$-Taylor method, we have the following balanced $\theta$-Taylor method:

$$
\begin{aligned}
x_{n+1}= & x_{n}+\Delta\left[(1-\theta) f\left(x_{n}\right)+\theta f\left(x_{n+1}\right)\right] \\
& +g\left(x_{n}\right) \Delta W_{n}+\frac{1}{2} g\left(x_{n}\right) g^{\prime}\left(x_{n}\right)\left[\left(\Delta W_{n}\right)^{2}-\Delta\right] \\
& +\frac{1}{2}\left(3 h\left(x_{n}\right)-h_{h}\left(x_{n}\right)\right) \Delta N_{n} \\
& +\left(g_{h}\left(x_{n}\right)-g\left(x_{n}\right)\right) \Delta W_{n} \Delta N_{n} \\
& +\frac{1}{2}\left(h_{h}\left(x_{n}\right)-h\left(x_{n}\right)\right)\left(\Delta N_{n}\right)^{2} \\
& +\left(g\left(x_{n}\right) h^{\prime}\left(x_{n}\right)-g_{h}\left(x_{n}\right)+g\left(x_{n}\right)\right) \Delta Z_{n} \\
& +C_{n}\left(x_{n}-x_{n+1}\right),
\end{aligned}
$$

where $C_{n}=C\left(x_{n}\right)=C_{0}\left(x_{n}\right) \Delta+C_{1}\left(x_{n}\right)\left[\left(\Delta W_{n}\right)^{2}-\Delta\right]$ with $C_{0}(\cdot)$ and $C_{1}(\cdot)$ called control functions.

\section{Convergence of the Implicit Taylor Methods}

Let $|\cdot|$ be the Euclidean norm in $\mathbb{R}^{d}$. If $A$ is a matrix, $|A|=$ $\sqrt{\operatorname{trace}\left(A^{T} A\right)}$. Denote $\|z\|_{L_{2}}=\left(\mathbb{E}|z|^{2}\right)^{1 / 2}$ for $z \in \mathbb{R}^{d}$. To prove the convergence of the numerical solutions, we make the following assumptions. 
Assumption 1. The coefficient functions $f, g$, and $h$ satisfy the global Lipschitz condition

$$
\begin{aligned}
& \left|f\left(x_{1}\right)-f\left(x_{2}\right)\right|+\left|g\left(x_{1}\right)-g\left(x_{2}\right)\right| \\
& \quad+\left|h\left(x_{1}\right)-h\left(x_{2}\right)\right| \\
& \quad+\left|g\left(x_{1}\right) g^{\prime}\left(x_{1}\right)-g\left(x_{2}\right) g^{\prime}\left(x_{2}\right)\right| \\
& \quad+\left|g\left(x_{1}\right) h^{\prime}\left(x_{1}\right)-g\left(x_{2}\right) h^{\prime}\left(x_{2}\right)\right| \leq L\left|x_{1}-x_{2}\right|
\end{aligned}
$$

for a positive constant $L$ and any $x_{1}, x_{2} \in \mathbb{R}^{d}$ and the linear growth condition

$$
\begin{aligned}
& |f(x)|^{2}+|g(x)|^{2}+|h(x)|^{2} \\
& \quad+\left|g(x) g^{\prime}(x)\right|^{2}+\left|g(x) h^{\prime}(x)\right|^{2} \leq L^{\prime}\left(1+|x|^{2}\right)
\end{aligned}
$$

for a positive constant $L^{\prime}$ and any $x \in \mathbb{R}^{d}$.

Assumption 2. The $C_{0}(\cdot)$ and $C_{1}(\cdot)$ are bounded $d \times d$-matrixvalued functions. For any real numbers $\alpha_{0} \in\left[0, \bar{\alpha}_{0}\right]$ and $\alpha_{1} \in$ $\left[-\bar{\alpha}_{1}, \bar{\alpha}_{1}\right]$ with $\bar{\alpha}_{0} \geq \Delta$ and $\bar{\alpha}_{1} \geq\left|\left(\Delta W_{n}\right)^{2}-\Delta\right|$ for all step-size $\Delta$ and $x \in \mathbb{R}^{d}$, the matrix $M(x)=I+\alpha_{0} C_{0}(x)+\alpha_{1} C_{1}(x)$ is reversible and satisfies $\left|(M(x))^{-1}\right| \leq B<\infty$, where $I$ is a unit matrix and $B$ is a positive constant.

In what follows, we will derive the strong convergence orders of the implicit Taylor methods for SDEJs (1).

\subsection{Convergence of the $\theta$-Taylor Method. Define}

$$
\begin{aligned}
\bar{x}^{\theta}\left(t_{n+1}\right)=x\left(t_{n}\right) & \\
+\Delta[ & (1-\theta) f\left(x\left(t_{n}\right)\right) \\
& \left.+\theta f\left(x\left(t_{n+1}\right)\right)\right]+g\left(x\left(t_{n}\right)\right) \Delta W_{n} \\
+ & \frac{1}{2} g\left(x\left(t_{n}\right)\right) g^{\prime}\left(x\left(t_{n}\right)\right)\left[\left(\Delta W_{n}\right)^{2}-\Delta\right] \\
+ & \frac{1}{2}\left(3 h\left(x\left(t_{n}\right)\right)-h_{h}\left(x\left(t_{n}\right)\right)\right) \Delta N_{n} \\
+ & \left(g_{h}\left(x\left(t_{n}\right)\right)-g\left(x\left(t_{n}\right)\right)\right) \Delta W_{n} \Delta N_{n} \\
+ & \frac{1}{2}\left(h_{h}\left(x\left(t_{n}\right)\right)-h\left(x\left(t_{n}\right)\right)\right)\left(\Delta N_{n}\right)^{2} \\
+ & \left(g\left(x\left(t_{n}\right)\right) h^{\prime}\left(x\left(t_{n}\right)\right)\right. \\
& \left.\quad-g_{h}\left(x\left(t_{n}\right)\right)+g\left(x\left(t_{n}\right)\right)\right) \Delta Z_{n}
\end{aligned}
$$

by replacing the numerical approximations with the exact solution values on the right-hand side of equation (3). Then, the local error of method (3) is defined by $\delta^{\theta}\left(t_{n+1}\right)=x\left(t_{n+1}\right)-$ $\bar{x}^{\theta}\left(t_{n+1}\right)$ and the global error of method (3) is defined by $\epsilon_{n}=x\left(t_{n}\right)-x_{n}$.

Theorem 3. Under Assumption 1, the $\theta$-Taylor method (3) is consistent with order 2 in the mean and with order 1.5 in the mean square. That is, the local mean error and mean-square error of the $\theta$-Taylor method (3) satisfy

$$
\begin{gathered}
\max _{0 \leq n \leq N-1}\left\|\mathbb{E}\left(\delta^{\theta}\left(t_{n+1}\right) \mid \mathscr{F}_{t_{n}}\right)\right\|_{L_{2}} \leq H_{1} \Delta^{2} \quad \text { as } \Delta \longrightarrow 0, \\
\max _{0 \leq n \leq N-1}\left\|\delta^{\theta}\left(t_{n+1}\right)\right\|_{L_{2}} \leq H_{2} \Delta^{3 / 2} \quad \text { as } \Delta \longrightarrow 0
\end{gathered}
$$

where the constants $H_{1}$ and $H_{2}$ are independent of $\Delta$.

Proof. To obtain the convergence rate of the $\theta$-Taylor method, we firstly introduce the local Taylor numerical approximation $\bar{x}_{n+1}^{A}$ which is defined by

$$
\begin{aligned}
\bar{x}_{n+1}^{A}=x & \left(t_{n}\right)+f\left(x\left(t_{n}\right)\right) \Delta+g\left(x\left(t_{n}\right)\right) \Delta W_{n} \\
+ & \frac{1}{2} g\left(x\left(t_{n}\right)\right) g^{\prime}\left(x\left(t_{n}\right)\right)\left[\left(\Delta W_{n}\right)^{2}-\Delta\right] \\
+ & \frac{1}{2}\left(3 h\left(x\left(t_{n}\right)\right)-h_{h}\left(x\left(t_{n}\right)\right)\right) \Delta N_{n} \\
+ & \left(g_{h}\left(x\left(t_{n}\right)\right)-g\left(x\left(t_{n}\right)\right)\right) \Delta W_{n} \Delta N_{n} \\
+ & \frac{1}{2}\left(h_{h}\left(x\left(t_{n}\right)\right)-h\left(x\left(t_{n}\right)\right)\right)\left(\Delta N_{n}\right)^{2} \\
+ & \left(g\left(x\left(t_{n}\right)\right) h^{\prime}\left(x\left(t_{n}\right)\right)-g_{h}\left(x\left(t_{n}\right)\right)\right. \\
& \left.+g\left(x\left(t_{n}\right)\right)\right) \Delta Z_{n} .
\end{aligned}
$$

Then, there exists some constant $K_{1}>0$ such that

$$
\begin{aligned}
\mathbb{E}\left[\left|\mathbb{E}\left[\left(\bar{x}_{n+1}^{\theta}-x\left(t_{n+1}\right)\right) \mid \mathscr{F}_{t_{n}}\right]\right|^{2}\right] \\
\leq 2 \mathbb{E}\left[\left|\mathbb{E}\left[\left(\bar{x}_{n+1}^{A}-x\left(t_{n+1}\right)\right) \mid \mathscr{F}_{t_{n}}\right]\right|^{2}\right] \\
+2 \mathbb{E}\left[\left|\mathbb{E}\left[\left(\bar{x}_{n+1}^{\theta}-\bar{x}_{n+1}^{A}\right) \mid \mathscr{F}_{t_{n}}\right]\right|^{2}\right] \\
\leq K_{1} \Delta^{4}+2 \mathbb{E}\left[\left|\mathbb{E}\left[\left(\bar{x}_{n+1}^{\theta}-\bar{x}_{n+1}^{A}\right) \mid \mathscr{F}_{t_{n}}\right]\right|^{2}\right] .
\end{aligned}
$$

Since

$$
\begin{aligned}
\mathbb{E}[\mid \mathbb{E} & {\left.\left.\left[\left(\bar{x}_{n+1}^{\theta}-\bar{x}_{n+1}^{A}\right) \mid \mathscr{F}_{t_{n}}\right]\right|^{2}\right] } \\
& =\mathbb{E}\left[\left|\mathbb{E}\left[\theta \Delta\left(f\left(x\left(t_{n+1}\right)\right)-f\left(x\left(t_{n}\right)\right)\right) \mid \mathscr{F}_{t_{n}}\right]\right|^{2}\right] \\
& \leq L \theta \Delta \mathbb{E}\left[\mathbb{E}\left[\left|x\left(t_{n+1}\right)-x\left(t_{n}\right)\right| \mid \mathscr{F}_{t_{n}}\right]\right]^{2} \\
& \leq O\left(\Delta^{4}\right),
\end{aligned}
$$

we obtain $\left(\mathbb{E}\left[\left|\mathbb{E}\left[\left(\bar{x}_{n+1}^{\theta}-x\left(t_{n+1}\right)\right) \mid \mathscr{F}_{t_{n}}\right]\right|^{2}\right]\right)^{1 / 2} \leq O\left(\Delta^{2}\right)$. 
On the other hand, since

$$
\begin{aligned}
& \mathbb{E}\left[\left|\bar{x}_{n+1}^{\theta}-\bar{x}_{n+1}^{A}\right|^{2} \mid x_{n}=x\left(t_{n}\right)\right] \\
& \quad=L^{2} \theta^{2} \Delta^{2} \mathbb{E}\left[\left|x\left(t_{n+1}\right)-x\left(t_{n}\right)\right|^{2}\right] \\
& \quad \leq O\left(\Delta^{3}\right),
\end{aligned}
$$

we have

$$
\begin{aligned}
& \mathbb{E}\left[\left|\bar{x}_{n+1}^{\theta}-x\left(t_{n+1}\right)\right|^{2}\right] \\
& \leq 2 \mathbb{E}\left[\left|\bar{x}_{n+1}^{A}-x\left(t_{n+1}\right)\right|^{2}\right] \\
& \quad+2 \mathbb{E}\left[\left|\bar{x}_{n+1}^{\theta}-\bar{x}_{n+1}^{A}\right|^{2}\right] \\
& \leq O\left(\Delta^{3}\right) .
\end{aligned}
$$

Therefore, the result (9) is obtained.

Theorem 4. Under Assumption 1, the $\theta$-Taylor method (3) is convergent with order 1 in the mean square. That is, the global error satisfies

$$
\max _{0 \leq n \leq N-1}\left\|\epsilon_{n+1}^{2}\right\|_{L_{2}} \leq H_{3} \Delta \quad \text { as } \Delta \longrightarrow 0
$$

where $\mathrm{H}_{3}$ is independent of $\Delta$.

Proof. From the definitions of $\delta_{n}$ and $\epsilon_{n}$, we have

$$
\epsilon_{n+1}=\epsilon_{n}+u_{n}+\delta_{n+1} \text {, }
$$

where

$$
\begin{aligned}
& u_{n}=\Delta(1-\theta)\left(f\left(x\left(t_{n}\right)\right)-f\left(x_{n}\right)\right) \\
& +\Delta \theta\left(f\left(x\left(t_{n+1}\right)\right)-f\left(x_{n+1}\right)\right) \\
& +\left(g\left(x\left(t_{n}\right)\right)-g\left(x_{n}\right)\right) \Delta W_{n} \\
& +\frac{1}{2}\left(g\left(x\left(t_{n}\right)\right) g^{\prime}\left(x\left(t_{n}\right)\right)\right. \\
& \left.\quad-g\left(x_{n}\right) g^{\prime}\left(x_{n}\right)\right)\left[(\Delta W)^{2}-\Delta\right] \\
& +\frac{1}{2}\left[3\left(h\left(x\left(t_{n}\right)\right)-h\left(x_{n}\right)\right)\right. \\
& \left.+\left(h_{h}\left(x\left(t_{n}\right)\right)-h_{h}\left(x_{n}\right)\right)\right] \Delta N_{n} \\
& +\left[\left(g_{h}\left(x\left(t_{n}\right)\right)-g_{h}\left(x_{n}\right)\right)\right. \\
& \left.\quad-\left(g\left(x\left(t_{n}\right)\right)-g\left(x_{n}\right)\right)\right] \Delta W_{n} \Delta N_{n} \\
& +\frac{1}{2}\left[\left(h_{h}\left(x\left(t_{n}\right)\right)-h_{h}\left(x_{n}\right)\right)\right. \\
& \left.+\left(h\left(x\left(t_{n}\right)\right)-h\left(x_{n}\right)\right)\right]\left(\Delta N_{n}\right)^{2} \\
& +\left[\left(g\left(x\left(t_{n}\right)\right) h^{\prime}\left(x\left(t_{n}\right)\right)-g\left(x_{n}\right) h^{\prime}\left(x_{n}\right)\right)\right. \\
& \left.-\left(g_{h}\left(x\left(t_{n}\right)\right)-g_{h}\left(x_{n}\right)\right)\right) \\
& \left.\left.+g\left(x_{n}\right)\right)\right] \Delta Z_{n} .
\end{aligned}
$$

Since $\epsilon_{n}$ is $\mathscr{F}_{t_{n}}$-measurable, we have from Theorem 3 that

$$
\begin{aligned}
& \left|\mathbb{E}\left\langle\delta_{n+1}, \epsilon_{n}\right\rangle\right| \\
& =\left|\mathbb{E}\left[\mathbb{E}\left(\left\langle\delta_{n+1}, \epsilon_{n}\right\rangle \mid \mathscr{F}_{t_{n}}\right)\right]\right| \\
& \leq \mathbb{E}\left|\left\langle\mathbb{E}\left(\delta_{n+1} \mid \mathscr{F}_{t_{n}}\right), \epsilon_{n}\right\rangle\right| \\
& \leq\left[\Delta^{-1}\left(\mathbb{E}\left|\mathbb{E}\left(\delta_{n+1} \mid \mathscr{F}_{t_{n}}\right)\right|^{2}\right)\right]^{1 / 2} \\
& \quad \times\left(\Delta \mathbb{E}\left|\epsilon_{n}\right|^{2}\right)^{1 / 2} \\
& \leq \Delta^{-1}\left(\mathbb{E}\left|\mathbb{E}\left(\delta_{n+1} \mid \mathscr{F}_{t_{n}}\right)\right|^{2}\right) \\
& \quad+\Delta \mathbb{E}\left|\epsilon_{n}\right|^{2} \\
& \leq H_{1} \Delta^{3}+\Delta \mathbb{E}\left|\epsilon_{n}\right|^{2}
\end{aligned}
$$

where $\langle\cdot, \cdot\rangle$ indicates the scalar product.

Noting that $\mathbb{E}\left|\Delta W_{n}\right|^{2}=\Delta, \mathbb{E}\left|\Delta W_{n}\right|^{4}=3 \Delta^{2}, \mathbb{E}\left(\Delta N_{n}\right)^{2}=$ $\lambda \Delta(1+\lambda \Delta), \mathbb{E}\left(\Delta N_{n}\right)^{4}=\lambda \Delta\left(1+7 \lambda \Delta+6(\lambda \Delta)^{2}+(\lambda \Delta)^{3}\right)$, and $\Delta W_{n}$ is independent of $\Delta N_{n}$, we have from Assumption 1 that

$$
\begin{aligned}
& \mathbb{E}\left|\left(g\left(x\left(t_{n}\right)\right)-g\left(x_{n}\right)\right) \Delta W_{n}\right|^{2} \\
& \leq \Delta \mathbb{E}\left|g\left(x\left(t_{n}\right)\right)-g\left(x_{n}\right)\right|^{2} \leq L^{2} \Delta \mathbb{E}\left|\epsilon_{n}\right|^{2} ; \\
& \mathbb{E}\left|\left(g\left(x\left(t_{n}\right)\right) g^{\prime}\left(x\left(t_{n}\right)\right)-g\left(x_{n}\right) g^{\prime}\left(x_{n}\right)\right)\left[(\Delta W)^{2}-\Delta\right]\right|^{2} \\
& \leq 2 \Delta^{2} \mathbb{E}\left|g\left(x\left(t_{n}\right)\right) g^{\prime}\left(x\left(t_{n}\right)\right)-g\left(x_{n}\right) g^{\prime}\left(x_{n}\right)\right| \\
& \leq 2 L^{2} \Delta^{2} \mathbb{E}\left|\epsilon_{n}\right|^{2} \\
& \mathbb{E}\left|\left[3\left(h\left(x\left(t_{n}\right)\right)-h\left(x_{n}\right)\right)-\left(h_{h}\left(x\left(t_{n}\right)\right)-h_{h}\left(x_{n}\right)\right)\right] \Delta N_{n}\right|^{2} \\
& \leq 2 \lambda \Delta(1+\lambda \Delta)\left[3 \mathbb{E}\left|h\left(x\left(t_{n}\right)\right)-h\left(x_{n}\right)\right|^{2}\right. \\
& \left.+\mathbb{E}\left|h\left(x\left(t_{n}\right)+h\left(x\left(t_{n}\right)\right)\right)-h\left(x_{n}+h\left(x_{n}\right)\right)\right|^{2}\right] \\
& \leq 2 L^{2} \lambda \Delta(1+\lambda \Delta)\left(5+2 L^{2}\right) \mathbb{E}\left|\epsilon_{n}\right|^{2} \\
& \mathbb{E}\left|\left[\left(g_{h}\left(x\left(t_{n}\right)\right)-g_{h}\left(x_{n}\right)\right)-\left(g\left(x\left(t_{n}\right)\right)-g\left(x_{n}\right)\right)\right] \Delta W_{n} \Delta N_{n}\right|^{2} \\
& \leq \mathbb{E}\left|\left(g_{h}\left(x\left(t_{n}\right)\right)-g_{h}\left(x_{n}\right)\right)-\left(g\left(x\left(t_{n}\right)\right)-g\left(x_{n}\right)\right)\right|^{2} \\
& \cdot \mathbb{E}\left|\Delta W_{n}\right|^{2} \cdot \mathbb{E}\left(\Delta N_{n}\right)^{2} \\
& \leq 2 L^{2} \lambda \Delta^{2}(1+\lambda \Delta)\left(3+2 L^{2}\right) \mathbb{E}\left|\epsilon_{n}\right|^{2} ; \\
& \mathbb{E}\left|\left[\left(h_{h}\left(x\left(t_{n}\right)\right)-h_{h}\left(x_{n}\right)\right)-\left(h\left(x\left(t_{n}\right)\right)-h\left(x_{n}\right)\right)\right]\left(\Delta N_{n}\right)^{2}\right|^{2} \\
& \leq 2 \lambda \Delta\left(1+7 \lambda \Delta+6(\lambda \Delta)^{2}+(\lambda \Delta)^{3}\right) L^{2}\left(3+2 L^{2}\right) \mathbb{E}\left|\epsilon_{n}\right|^{2} ; \\
& \mathbb{E} \mid\left[\left(g\left(x\left(t_{n}\right)\right) h^{\prime}\left(x\left(t_{n}\right)\right)-g\left(x_{n}\right) h^{\prime}\left(x_{n}\right)\right)\right. \\
& \left.-\left(g_{h}\left(x\left(t_{n}\right)\right)-g_{h}\left(x_{n}\right)\right)+\left(g\left(x\left(t_{n}\right)\right)-g\left(x_{n}\right)\right)\right]\left.\Delta Z_{n}\right|^{2}
\end{aligned}
$$




$$
\begin{aligned}
& \leq 6 L^{2}\left(2+L^{2}\right) \mathbb{E}\left|\epsilon_{n}\right|^{2} \cdot \mathbb{E}\left|\Delta Z_{n}\right|^{2} \\
& \leq 6 L^{2}\left(2+L^{2}\right) \mathbb{E}\left|\epsilon_{n}\right|^{2} \cdot \mathbb{E}\left|\Delta W_{n}-\Delta Z_{1 n}\left(t_{n+1}\right)\right|^{2} \\
& \leq 24 L^{2}\left(2+L^{2}\right) \Delta \mathbb{E}\left|\epsilon_{n}\right|^{2} .
\end{aligned}
$$

Hence,

$$
\mathbb{E}\left|u_{n}\right|^{2} \leq J_{1} \Delta \mathbb{E}\left|\epsilon_{n}\right|^{2}+8 L^{2} \Delta^{2} \theta^{2} \mathbb{E}\left|\epsilon_{n+1}\right|^{2},
$$

where $J_{1}=8\left[4 L^{2}+2 L^{2} \lambda(1+\lambda)\left(5+2 L^{2}\right)+2 L^{2} \lambda(1+\lambda)(3+\right.$ $\left.2 L^{2}\right)+2 \lambda\left(1+7 \lambda+6 \lambda^{2}+\lambda^{3}\right) L^{2}\left(3+2 L^{2}\right)+24 L^{2}\left(2+L^{2}\right)$.

Noting that $x\left(t_{n}\right)$ and $x_{n}$ are $\mathscr{F}_{t_{n}}$-measurable and $\Delta W_{n}$ and $\Delta N_{n}$ are independent of $\mathscr{F}_{t_{n}}$, we have

$$
\begin{gathered}
\mathbb{E}\left[\left(g\left(x\left(t_{n}\right)\right)-g\left(x_{n}\right)\right) \Delta W_{n} \mathscr{F}_{t_{n}}\right]=0 ; \\
\mathbb{E}\left[\left(g\left(x\left(t_{n}\right)\right) g^{\prime}\left(x\left(t_{n}\right)\right)-g\left(x_{n}\right) g^{\prime}\left(x_{n}\right)\right)\right. \\
\left.\times\left((\Delta W)^{2}-\Delta\right) \mid \mathscr{F}_{t_{n}}\right]=0 ; \\
\mathbb{E}\left[\left[\left(g_{h}\left(x\left(t_{n}\right)\right)-g_{h}\left(x_{n}\right)\right)-\left(g\left(x\left(t_{n}\right)\right)-g\left(x_{n}\right)\right)\right]\right. \\
\left.\times \Delta W_{n} \Delta N_{n} \mid \mathscr{F}_{t_{n}}\right]=0 ; \\
\mathbb{E}\left[\left[\left(g\left(x\left(t_{n}\right)\right) h^{\prime}\left(x\left(t_{n}\right)\right)-g\left(x_{n}\right) h^{\prime}\left(x_{n}\right)\right)\right.\right. \\
-\left(g_{h}\left(x\left(t_{n}\right)\right)-g_{h}\left(x_{n}\right)\right) \\
\left.\left.+\left(g\left(x\left(t_{n}\right)\right)-g\left(x_{n}\right)\right)\right] \Delta Z_{n} \mid \mathscr{F}_{t_{n}}\right]=0 .
\end{gathered}
$$

Therefore,

$$
\begin{aligned}
\left|\mathbb{E}\left(u_{n} \mid \mathscr{F}_{t_{n}}\right)\right|^{2} & \leq \mid \Delta(1-\theta) \mathbb{E}\left[f\left(x\left(t_{n}\right)\right)-f\left(x_{n}\right) \mid \mathscr{F}_{t_{n}}\right] \\
& +\theta \Delta \mathbb{E}\left[f\left(x\left(t_{n+1}\right)\right)-f\left(x_{n+1}\right) \mid \mathscr{F}_{t_{n}}\right] \\
& +\frac{3}{2} \mathbb{E}\left[\left(h\left(x\left(t_{n}\right)\right)-h\left(x_{n}\right)\right) \Delta N_{n} \mid \mathscr{F}_{t_{n}}\right] \\
& -\frac{1}{2} \mathbb{E}\left[\left(h_{h}\left(x\left(t_{n}\right)\right)-h_{h}\left(x_{n}\right)\right) \Delta N_{n} \mid \mathscr{F}_{t_{n}}\right] \\
& +\frac{1}{2} \mathbb{E}\left[\left(h_{h}\left(x\left(t_{n}\right)\right)-h_{h}\left(x_{n}\right)\right)\left(\Delta N_{n}\right)^{2} \mid \mathscr{F}_{t_{n}}\right] \\
& -\left.\frac{1}{2} \mathbb{E}\left[\left(h\left(x\left(t_{n}\right)\right)-h\left(x_{n}\right)\right)\left(\Delta N_{n}\right)^{2} \mid \mathscr{F}_{t_{n}}\right]\right|^{2} \\
\leq & J_{2} \Delta^{2} \mathbb{E}\left|\epsilon_{n}\right|^{2}+6 L^{2} \theta^{2} \Delta^{2} \mathbb{E}\left[\left|\epsilon_{n+1}\right|^{2} \mid \mathscr{F}_{t_{n}}\right],
\end{aligned}
$$

where $J_{2}=3 L^{2}\left[2+3 \lambda^{2}+(1+L)^{2} \lambda^{2}+(1+L)^{2}(1+\lambda)^{2}+\right.$ $\left.\lambda^{2}(1+\lambda)^{2}\right]$. Thus,

$$
\begin{aligned}
\left|\mathbb{E}\left\langle\epsilon_{n}, u_{n}\right\rangle\right| & \leq \Delta^{-1}\left(\mathbb{E}\left|\mathbb{E}\left(u_{n} \mid \mathscr{F}_{t_{n}}\right)\right|^{2}\right)+\Delta \mathbb{E}\left|\epsilon_{n}\right|^{2} \\
& \leq\left(1+J_{2}\right) \Delta \mathbb{E}\left|\epsilon_{n}\right|^{2}+6 L^{2} \theta^{2} \Delta \mathbb{E}\left|\epsilon_{n+1}\right|^{2} .
\end{aligned}
$$

From the above arguments, we obtain

$$
\begin{aligned}
\mathbb{E}\left|\epsilon_{n+1}\right|^{2} & \\
\leq & \mathbb{E}\left|\epsilon_{n}\right|^{2}+\mathbb{E}\left|\delta_{n+1}\right|^{2}+\mathbb{E}\left|u_{n}\right|^{2} \\
& +2\left|\mathbb{E}\left\langle\delta_{n+1}, \epsilon_{n}\right\rangle\right|+2\left|\mathbb{E}\left\langle\epsilon_{n}, u_{n}\right\rangle\right| \\
\leq & {\left[1+2\left(2+J_{1}+J_{2}\right) \Delta\right] \mathbb{E}\left|\epsilon_{n}\right|^{2} } \\
& +2 L^{2} \theta^{2} \Delta(\Delta+6) \mathbb{E}\left|\epsilon_{n+1}\right|^{2} \\
& +\left(H_{2}+2 H_{1}\right) \Delta^{3} .
\end{aligned}
$$

Because $\Delta \rightarrow 0$, we can assume $1-2 L^{2} \theta^{2} \Delta(\Delta+6)>0$ without loss of generality. Let $J_{3}=2\left(2+J_{1}+J_{2}\right)$. Then,

$$
\begin{aligned}
\mathbb{E}\left|\epsilon_{n+1}\right|^{2} & \leq\left(1+J_{3} \Delta\right) \mathbb{E}\left|\epsilon_{n}\right|^{2}+\left(H_{2}+2 H_{1}\right) \Delta^{3} \\
= & \left(H_{2}+2 H_{1}\right) \Delta^{2} \frac{\left(1+J_{3} \Delta\right)^{n+1}-1}{J_{3}} \leq J_{4} \Delta^{2},
\end{aligned}
$$

where $J_{4}=\left(H_{2}+2 H_{1}\right)\left(\left(e^{J_{3} T}-1\right) / J_{3}\right)$.

\subsection{Convergence of the Balanced $\theta$-Taylor Method. Define}

$$
\begin{aligned}
\bar{x}^{B}\left(t_{n+1}\right) & \\
= & x\left(t_{n}\right)+\Delta\left[(1-\theta) f\left(x\left(t_{n}\right)\right)+\theta f\left(x\left(t_{n+1}\right)\right)\right] \\
& +g\left(x\left(t_{n}\right)\right) \Delta W_{n} \\
& +\frac{1}{2} g\left(x\left(t_{n}\right)\right) g^{\prime}\left(x\left(t_{n}\right)\right)\left[\left(\Delta W_{n}\right)^{2}-\Delta\right] \\
+ & \frac{1}{2}\left(3 h\left(x\left(t_{n}\right)\right)-h_{h}\left(x\left(t_{n}\right)\right)\right) \Delta N_{n} \\
+ & \left(g_{h}\left(x\left(t_{n}\right)\right)-g\left(x\left(t_{n}\right)\right)\right) \Delta W_{n} \Delta N_{n} \\
+ & \frac{1}{2}\left(h_{h}\left(x\left(t_{n}\right)\right)-h\left(x\left(t_{n}\right)\right)\right)\left(\Delta N_{n}\right)^{2} \\
+ & \left(g\left(x\left(t_{n}\right)\right) h^{\prime}\left(x\left(t_{n}\right)\right)-g_{h}\left(x\left(t_{n}\right)\right)\right. \\
& \left.\quad+g\left(x\left(t_{n}\right)\right)\right) \Delta Z_{n}+C_{n}\left(x\left(t_{n}\right)-\bar{x}^{B}\left(t_{n+1}\right)\right)
\end{aligned}
$$

by replacing the numerical approximations with the exact solution values on the right-hand side of (4). Then, the local error of method (4) is $\delta^{B}\left(t_{n+1}\right)=x\left(t_{n+1}\right)-\bar{x}^{B}\left(t_{n+1}\right)$ and the global error of method (4) is $\epsilon_{n}=x\left(t_{n}\right)-x_{n}$.

Theorem 5. Under Assumptions 1 and 2, the balanced $\theta$-Taylor method (4) is consistent with order 2 in the mean and with 
order 1.5 in the mean square. That is, the local mean error and mean-square error of the balanced $\theta$-Taylor method (4) satisfy

$$
\begin{gathered}
\max _{0 \leq n \leq N-1}\left\|\mathbb{E}\left(\delta^{B}\left(t_{n+1}\right) \mid \mathscr{F}_{t_{n}}\right)\right\|_{L_{2}} \leq H_{4} \Delta^{2} \quad \text { as } \Delta \longrightarrow 0, \\
\max _{0 \leq n \leq N-1}\left\|\delta^{B}\left(t_{n+1}\right)\right\|_{L_{2}} \leq H_{5} \Delta^{3 / 2} \quad \text { as } \Delta \longrightarrow 0,
\end{gathered}
$$

where the constants $H_{4}$ and $H_{5}$ are independent of $\Delta$.

Proof. From Theorem 3, we have

$$
\begin{aligned}
& \mathbb{E}\left[\left|\mathbb{E}\left[\left(\bar{x}_{n+1}^{B}-x\left(t_{n+1}\right)\right) \mid \mathscr{F}_{t_{n}}\right]\right|^{2}\right] \\
& \leq 2 \mathbb{E}\left[\left|\mathbb{E}\left[\left(\bar{x}_{n+1}^{\theta}-x\left(t_{n+1}\right)\right) \mid \mathscr{F}_{t_{n}}\right]\right|^{2}\right] \\
& +2 \mathbb{E}\left[\left|\mathbb{E}\left[\left(\bar{x}_{n+1}^{B}-\bar{x}_{n+1}^{\theta}\right) \mid \mathscr{F}_{t_{n}}\right]\right|^{2}\right] \\
& \leq H_{1}^{2} \Delta^{4}+2 \mathbb{E}\left[\left|\mathbb{E}\left[\left(\bar{x}_{n+1}^{B}-\bar{x}_{n+1}^{\theta}\right) \mid \mathscr{F}_{t_{n}}\right]\right|^{2}\right] .
\end{aligned}
$$

From the definitions of $\bar{x}_{n+1}^{\theta}$ and $\bar{x}_{n+1}^{B}$ in (7) and (26), we can write

$$
\begin{aligned}
\bar{x}_{n+1}^{B}-\bar{x}_{n+1}^{\theta} & \\
=-C_{n} & \left(x\left(t_{n}\right)-\bar{x}^{B}\left(t_{n+1}\right)\right) \\
=C_{n}[ & \Delta\left[(1-\theta) f\left(x\left(t_{n}\right)\right)+\theta f\left(x\left(t_{n+1}\right)\right)\right] \\
+ & g\left(x\left(t_{n}\right)\right) \Delta W_{n} \\
+ & \frac{1}{2} g\left(x\left(t_{n}\right)\right) g^{\prime}\left(x\left(t_{n}\right)\right)\left[\left(\Delta W_{n}\right)^{2}-\Delta\right] \\
+ & \frac{1}{2}\left(3 h\left(x\left(t_{n}\right)\right)-h_{h}\left(x\left(t_{n}\right)\right)\right) \Delta N_{n} \\
+ & \left(g_{h}\left(x\left(t_{n}\right)\right)-g\left(x\left(t_{n}\right)\right)\right) \Delta W_{n} \Delta N_{n} \\
+ & \frac{1}{2}\left(h_{h}\left(x\left(t_{n}\right)\right)-h\left(x\left(t_{n}\right)\right)\right)\left(\Delta N_{n}\right)^{2} \\
+ & \left(g\left(x\left(t_{n}\right)\right) h^{\prime}\left(x\left(t_{n}\right)\right)-g_{h}\left(x\left(t_{n}\right)\right)\right. \\
& \left.\left.+g\left(x\left(t_{n}\right)\right)\right) \Delta Z_{n}+C_{n}\left(x_{n}-x_{n+1}^{B}\right)\right] \\
=(I+ & \left.C_{n}\right)^{-1} C_{n} \\
\times[\Delta & {\left[(1-\theta) f\left(x\left(t_{n}\right)\right)+\theta f\left(x\left(t_{n+1}\right)\right)\right] } \\
& +g\left(x\left(t_{n}\right)\right) \Delta W_{n}+\frac{1}{2} g\left(x\left(t_{n}\right)\right) \\
& \times g^{\prime}\left(x\left(t_{n}\right)\right)\left[\left(\Delta W_{n}\right)^{2}-\Delta\right] \\
& +\frac{1}{2}\left(3 h\left(x\left(t_{n}\right)\right)-h_{h}\left(x\left(t_{n}\right)\right)\right) \Delta N_{n} \\
& +\left(g_{h}\left(x\left(t_{n}\right)\right)-g\left(x\left(t_{n}\right)\right)\right) \Delta W_{n} \Delta N_{n} \\
& \frac{1}{2}\left(h_{h}\left(x\left(t_{n}\right)\right)-h\left(x\left(t_{n}\right)\right)\right)\left(\Delta N_{n}\right)^{2}
\end{aligned}
$$

$$
\begin{aligned}
& +\left(g\left(x\left(t_{n}\right)\right) h^{\prime}\left(x\left(t_{n}\right)\right)-g_{h}\left(x\left(t_{n}\right)\right)\right. \\
& \left.\left.+g\left(x\left(t_{n}\right)\right)\right) \Delta Z_{n}\right] .
\end{aligned}
$$

Since the components of the matrices $C_{0}(\cdot)$ and $C_{1}(\cdot)$ in $C_{n}(\cdot)$ are bounded, there exists a positive constant $M$ such that $\left|C_{i}\right| \leq M(i=0,1)$. Under Assumptions 1 and 2, we have

$$
\begin{aligned}
& \left|\mathbb{E}\left[\left(I+C_{n}\right)^{-1} C_{n} \Delta f\left(x\left(t_{n}\right)\right) \mid \mathscr{F}_{t_{n}}\right]\right| \\
& \leq M \Delta\left|f\left(x_{n}\right)\right| \mathbb{E}\left[\left|C_{0} \Delta+C_{1}\left(\left(\Delta W_{n}\right)^{2}-\Delta\right)\right| \mid \mathscr{F}_{t_{n}}\right] \\
& \leq L^{\prime} M B\left(1+\left|x\left(t_{n}\right)\right|^{2}\right)^{1 / 2} \Delta^{2} \text {; } \\
& \left|\mathbb{E}\left[\left(I+C_{n}\right)^{-1} C_{n} g\left(x\left(t_{n}\right)\right) \Delta W_{n} \mid \mathscr{F}_{t_{n}}\right]\right|=0 ; \\
& \mid \mathbb{E}\left[\left(I+C_{n}\right)^{-1} C_{n} g\left(x\left(t_{n}\right)\right) g^{\prime}\left(x\left(t_{n}\right)\right)\right. \\
& \left.\times\left[\left(\Delta W_{n}\right)^{2}-\Delta\right] \mid \mathscr{F}_{t_{n}}\right] \mid \\
& \leq B M\left|g\left(x\left(t_{n}\right)\right) g^{\prime}\left(x\left(t_{n}\right)\right)\right| \\
& \mathbb{E}\left[\left|\left(\left(\Delta W_{n}\right)^{2}-\Delta\right)^{2}\right| \mid \mathscr{F}_{t_{n}}\right] \\
& \leq 2 L^{\prime} M B\left(1+\left|x_{n}\right|^{2}\right)^{1 / 2} \Delta^{2} ; \\
& \left|\left[\mathbb{E}\left[\left(I+C_{n}\right)^{-1} C_{n}\left(3 h\left(x\left(t_{n}\right)\right)-h_{h}\left(x\left(t_{n}\right)\right)\right) \Delta N_{n}\right] \mid \mathscr{F}_{t_{n}}\right]\right| \\
& \leq \lambda B M\left|3 h\left(x\left(t_{n}\right)\right)-h_{h}\left(x\left(t_{n}\right)\right)\right| \Delta^{2} \\
& \leq(2+L) L^{\prime} B M\left(1+\left|x\left(t_{n}\right)\right|^{2}\right)^{1 / 2} \Delta^{2}, \\
& \mid \mathbb{E}\left[\left(I+C_{n}\right)^{-1} C_{n}\left(g_{h}\left(x\left(t_{n}\right)\right)-g\left(x\left(t_{n}\right)\right)\right)\right. \\
& \left.\times \Delta W_{n} \Delta N_{n} \mid \mathscr{F}_{t_{n}}\right] \mid=0 ; \\
& \mid \mathbb{E}\left[\left(I+C_{n}\right)^{-1} C_{n}\left(h_{h}\left(x\left(t_{n}\right)\right)-h\left(x\left(t_{n}\right)\right)\right)\right. \\
& \left.\times\left(\Delta N_{n}\right)^{2} \mid \mathscr{F}_{t_{n}}\right] \mid \\
& \leq B M\left|h_{h}\left(x\left(t_{n}\right)\right)-h\left(x\left(t_{n}\right)\right)\right| \lambda \Delta^{2}(1+\lambda \Delta) \\
& \leq L^{\prime} B M \lambda\left(1+\left|x\left(t_{n}\right)\right|^{2}\right)^{1 / 2} \Delta^{2}(1+\lambda \Delta) ; \\
& \mid \mathbb{E}\left[( I + C _ { n } ) ^ { - 1 } C _ { n } \left(g\left(x\left(t_{n}\right)\right) h^{\prime}\left(x\left(t_{n}\right)\right)\right.\right. \\
& \left.\left.-g_{h}\left(x\left(t_{n}\right)\right)+g\left(x\left(t_{n}\right)\right)\right) \Delta Z_{n} \mid \mathscr{F}_{t_{n}}\right] \mid \\
& =\mid \mathbb{E}\left[( I + C _ { n } ) ^ { - 1 } C _ { n } \left(g\left(x\left(t_{n}\right)\right) h^{\prime}\left(x\left(t_{n}\right)\right)\right.\right. \\
& \left.-g_{h}\left(x\left(t_{n}\right)\right)+g\left(x\left(t_{n}\right)\right)\right) \\
& \left.\times\left(\Delta W_{n}-\Delta Z_{1 k}\left(t_{n+1}\right)\right) \mid \mathscr{F}_{t_{n}}\right] \mid \\
& =0 \text {. }
\end{aligned}
$$


Therefore,

$$
\mathbb{E}\left[\left|\mathbb{E}\left[\left(\bar{x}_{n+1}^{B}-x\left(t_{n+1}\right)\right) \mid \mathscr{F}_{t_{n}}\right]\right|^{2}\right] \leq O\left(\Delta^{4}\right) .
$$

On the other hand, since

$$
\begin{aligned}
\mathbb{E}\left[\left|\left(I+C_{n}\right)^{-1} C_{n} \Delta\left[(1-\theta) f\left(x\left(t_{n}\right)\right)+\theta f\left(x\left(t_{n+1}\right)\right)\right]\right|^{2}\right] \\
\leq O\left(\Delta^{4}\right) ; \\
\mathbb{E}\left[\left|\left(I+C_{n}\right)^{-1} C_{n} g\left(x_{n}\right) \Delta W_{n}\right|^{2}\right] \leq O\left(\Delta^{3}\right) ; \\
\mathbb{E}\left[\left|\left(I+C_{n}\right)^{-1} C_{n} g\left(x\left(t_{n}\right)\right) g^{\prime}\left(x\left(t_{n}\right)\right)\left[\left(\Delta W_{n}\right)^{2}-\Delta\right]\right|^{2}\right] \\
\leq O\left(\Delta^{3}\right) ; \\
\mathbb{E}\left[\left|\left(I+C_{n}\right)^{-1} C_{n}\left(3 h\left(x\left(t_{n}\right)\right)-h_{h}\left(x\left(t_{n}\right)\right)\right) \Delta N_{n}\right|^{2}\right] \\
\leq O\left(\Delta^{3}\right) ; \\
\mathbb{E}\left[\left|\left(I+C_{n}\right)^{-1} C_{n}\left(g_{h}\left(x\left(t_{n}\right)\right)-g\left(x\left(t_{n}\right)\right)\right) \Delta W_{n} \Delta N_{n}\right|^{2}\right] \\
\leq O\left(\Delta^{4}\right) ; \\
\mathbb{E}\left[\left|\left(I+C_{n}\right)^{-1} C_{n}\left(h_{h}\left(x\left(t_{n}\right)\right)-h\left(x\left(t_{n}\right)\right)\right)\left(\Delta N_{n}\right)^{2}\right|^{2}\right] \\
\leq O\left(\Delta^{3}\right) ; \\
\mathbb{E}\left[\mid\left(I+C_{n}\right)^{-1} C_{n}\left(g\left(x\left(t_{n}\right)\right) h^{\prime}\left(x\left(t_{n}\right)\right)-g_{h}\left(x\left(t_{n}\right)\right)\right.\right. \\
\quad \leq O\left(\Delta^{3}\right),
\end{aligned}
$$

we have

$$
\begin{aligned}
& \mathbb{E}\left[\left|x_{n+1}^{B}-x\left(t_{n+1}\right)\right|^{2}\right] \\
& \leq 2 \mathbb{E}\left[\left|x\left(t_{n+1}\right)-x_{n+1}^{\theta}\right|^{2}\right]+2 \mathbb{E}\left[\left|x_{n+1}^{\theta}-x_{n+1}^{B}\right|^{2}\right] \\
& \leq O\left(\Delta^{3}\right) .
\end{aligned}
$$

Theorem 6. Under Assumptions 1 and 2, the balanced $\theta$ Taylor method (4) is convergent with order 1 in the mean square. That is, the global error satisfies

$$
\max _{0 \leq n \leq N-1}\left\|\epsilon_{n+1}^{2}\right\|_{L_{2}} \leq H_{6} \Delta \quad \text { as } \Delta \longrightarrow 0,
$$

where $\mathrm{H}_{6}$ is independent of $\Delta$.

Proof. From the definitions of $\delta_{n}$ and $\epsilon_{n}$, we have

$$
\epsilon_{n+1}=\epsilon_{n}+P_{n}+\delta_{n+1} \text {, }
$$

where

$$
\begin{aligned}
P_{n}=u_{n} & +C\left(x\left(t_{n}\right)\right)\left(x\left(t_{n}\right)-\bar{x}^{B}\left(t_{n+1}\right)\right) \\
& -C\left(x_{n}\right)\left(x_{n}-x_{n+1}\right) \\
=u_{n} & +C\left(x\left(t_{n}\right)\right)\left(x\left(t_{n}\right)-x_{n}\right) \\
& -C\left(x\left(t_{n}\right)\right)\left(\bar{x}^{B}\left(t_{n+1}\right)-x_{n+1}\right) \\
& +\left(C\left(x\left(t_{n}\right)\right)-C\left(x_{n}\right)\right)\left(x_{n}-x_{n+1}\right) \\
=u_{n} & -C\left(x\left(t_{n}\right)\right) P_{n} \\
& +\left(C\left(x\left(t_{n}\right)\right)-C\left(x_{n}\right)\right)\left(x_{n}-x_{n+1}\right) \\
=( & \left.+C\left(x\left(t_{n}\right)\right)\right)^{-1} \\
& \times\left[u_{n}+\left(C\left(x\left(t_{n}\right)\right)-C\left(x_{n}\right)\right)\left(x_{n}-x_{n+1}\right)\right] .
\end{aligned}
$$

Thus, there exists a constant $J_{5}$ such that

$$
\begin{aligned}
\mathbb{E}\left|P_{n}\right|^{2} & \\
\leq & 2 B^{2} \mathbb{E}\left|u_{n}\right|^{2}+2 B^{2} \mathbb{E} \mid\left[\left(C_{0}\left(x\left(t_{n}\right)\right)-C_{0}\left(x_{n}\right)\right) \Delta\right. \\
& \left.+\left(C_{1}\left(x\left(t_{n}\right)\right)-C_{1}\left(x_{n}\right)\right)\left(\left(\Delta W_{n}\right)^{2}-\Delta\right)\right]\left.\left(x_{n}-x_{n+1}\right)\right|^{2} \\
\leq & 2 B^{2} \mathbb{E}\left|u_{n}\right|^{2}+4 B^{2} M^{2} \Delta^{2} \mathbb{E}\left|\left(x_{n}-x_{n+1}\right)\right|^{2} \\
\leq & 2 B^{2} J_{1} \Delta \mathbb{E}\left|\epsilon_{n}\right|^{2} \\
& +2 B^{2} L^{2} \Delta^{2} \theta^{2} \mathbb{E}\left|\epsilon_{n+1}\right|^{2}+J_{5} \Delta^{3}
\end{aligned}
$$

and there exists a constant $J_{6}$ such that

$$
\begin{aligned}
\left|\mathbb{E}\left(P_{n} \mid \mathscr{F}_{t_{n}}\right)\right|^{2} & \\
= & \mid \mathbb{E}\left(\left(I+C\left(x\left(t_{n}\right)\right)\right)^{-1}\right. \\
& \quad\left[u_{n}+\left(C\left(x\left(t_{n}\right)\right)-C\left(x_{n}\right)\right)\right. \\
& \left.\left.\times\left(x_{n}-x_{n+1}\right)\right] \mid \mathscr{F}_{t_{n}}\right)\left.\right|^{2} \\
\leq & 2 B^{2}\left|\mathbb{E}\left(u_{n} \mid \mathscr{F}_{t_{n}}\right)\right|^{2}+2 B^{2} \\
& \times\left|\mathbb{E}\left(\left(C\left(x\left(t_{n}\right)\right)-C\left(x_{n}\right)\right)\left(x_{n}-x_{n+1}\right) \mid \mathscr{F}_{t_{n}}\right)\right|^{2} \\
\leq & 2 B^{2}\left(J_{2} \Delta^{2} \mathbb{E}\left|\epsilon_{n}\right|^{2}+L^{2} \Delta^{2} \theta^{2} \mathbb{E}\left(\left|\epsilon_{n+1}\right|^{2} \mid \mathscr{F}_{t_{n}}\right)\right) \\
+ & 2 B^{2} \Delta\left|\mathbb{E}\left(\left(C_{0}\left(x\left(t_{n}\right)\right)-C_{0}\left(x_{n}\right)\right)\left(x_{n}-x_{n+1}\right) \mid \mathscr{F}_{t_{n}}\right)\right|^{2} \\
\leq & 2 B^{2} J_{2} \Delta^{2} \mathbb{E}\left|\epsilon_{n}\right|^{2} \\
& +2 B^{2} L^{2} \Delta^{2} \theta^{2} \mathbb{E}\left(\left|\epsilon_{n+1}\right|^{2} \mid \mathscr{F}_{t_{n}}\right)+J_{6} \Delta^{4} .
\end{aligned}
$$


Thus,

$$
\begin{aligned}
&\left|\mathbb{E}\left\langle\epsilon_{n}, P_{n}\right\rangle\right| \\
& \leq \Delta^{-1}\left(\mathbb{E}\left|\mathbb{E}\left(P_{n} \mid \mathscr{F}_{t_{n}}\right)\right|^{2}\right)+\Delta \mathbb{E}\left|\epsilon_{n}\right|^{2} \\
& \leq\left(1+2 B^{2} J_{2}\right) \Delta \mathbb{E}\left|\epsilon_{n}\right|^{2} \\
&+12 B^{2} L^{2} \theta^{2} \Delta \mathbb{E}\left|\epsilon_{n+1}\right|^{2}+J_{6} \Delta^{3} .
\end{aligned}
$$

From Theorem 5, we have

$$
\left|\mathbb{E}\left\langle\delta_{n+1}, \epsilon_{n}\right\rangle\right| \leq H_{4} \Delta^{3}+\Delta \mathbb{E}\left|\epsilon_{n}\right|^{2} .
$$

Therefore,

$$
\begin{aligned}
\mathbb{E}\left|\epsilon_{n+1}\right|^{2} & \\
\leq & \left(1+J_{7} \Delta\right) \mathbb{E}\left|\epsilon_{n}\right|^{2} \\
& +2 B^{2} L^{2} \theta^{2} \Delta(\Delta+12) \mathbb{E}\left|\epsilon_{n+1}\right|^{2} \\
& +\left(J_{5}+H_{5}+2 H_{4}+2 J_{6}\right) \Delta^{3}
\end{aligned}
$$

where $J_{7}=2\left(B^{2} J_{1}+2+2 B^{2} J_{2}\right)$. Because $\Delta \rightarrow 0$, we can assume $1-2 B^{2} L^{2} \theta^{2} \Delta(\Delta+12)>0$ without loss of generality. Let $J_{8}=J_{5}+H_{5}+2 H_{4}+2 J_{6}$. Then,

$$
\begin{aligned}
\mathbb{E}\left|\epsilon_{n+1}\right|^{2} & \leq\left(1+J_{7} \Delta\right) \mathbb{E}\left|\epsilon_{n}\right|^{2}+J_{8} \Delta^{3} \\
& =J_{8} \Delta^{2} \frac{\left(1+J_{7} \Delta\right)^{n+1}-1}{J_{7}} \leq J_{9} \Delta^{2},
\end{aligned}
$$

where $J_{9}=J_{8}\left(\left(e^{J_{7} T}-1\right) / J_{7}\right)$.

\section{Stability of the Implicit Taylor Methods}

In this section, we will discuss the stability properties of the numerical methods introduced in Section 2. Consider a scalar linear test equation,

$$
\begin{array}{r}
\mathrm{d} x(t)=a x(t) \mathrm{d} t+b x(t) \mathrm{d} W(t)+c x(t) \mathrm{d} N(t), \\
x\left(t_{0}\right)=x_{0},
\end{array}
$$

where $a, b$, and $c$ are real constants. The solution of (43) is $x(t)=x_{0} e^{\left(a-(1 / 2) b^{2}\right) t+b W(t)}(1+c)^{N(t)}$ and is mean-square (MS) stable if $2 a+b^{2}+\lambda c(2+c)<0[2]$.

The one-step scheme of the test equation (43) is

$$
x_{n+1}=R\left(a, b, c, \Delta, \Delta W_{n}, \Delta N_{n}\right) x_{n} .
$$

The numerical method is MS-stable if

$$
\bar{R}(a, b, c, \Delta, \lambda)=\mathbb{E}\left(R^{2}\left(a, b, c, \Delta, \Delta W_{n}, \Delta N_{n}\right)\right)<1,
$$

where $\bar{R}(a, b, c, \Delta, \lambda)$ is called the MS-stability function of the numerical method.

If the Taylor method (2) is applied to the test equation (43), we obtain

$$
x_{n+1}=R_{1}\left(a, b, c, \Delta, \Delta W_{n}, \Delta N_{n}\right) x_{n},
$$

where

$$
\begin{aligned}
R_{1}\left(a, b, c, \Delta, \Delta W_{n}, \Delta N_{n}\right) \\
=1+\left(a-\frac{1}{2} b^{2}\right) \Delta+b \Delta W_{n}+\frac{1}{2} b^{2}\left(\Delta W_{n}\right)^{2} \\
+\frac{1}{2}\left(2 c-c^{2}\right) \Delta N_{n}+b c \Delta W_{n} \Delta N_{n} \\
+\frac{1}{2} c^{2}\left(\Delta N_{n}\right)^{2} .
\end{aligned}
$$

Let $p=a \Delta, q=b \sqrt{\Delta}$, and $z=c \lambda \Delta$. Then the MS-stability function of the Taylor method is

$$
\begin{aligned}
\bar{R}_{1}(p, q, z, c) \\
=\mathbb{E}\left(R_{1}^{2}\left(a, b, c, \Delta, \Delta W_{n}, \Delta N_{n}\right)\right) \\
=1+2 p+q^{2}+p^{2}+\frac{1}{2} p q^{2}+\frac{1}{2} q^{4} \\
+\left(2+c+2 p+c q^{2}+2 q^{2}\right) z \\
+\left(2+2 c+\frac{1}{2} c^{2}+q^{2}+p\right) z^{2} \\
+(c+1) z^{3}+\frac{1}{4} z^{4} .
\end{aligned}
$$

Thus, the strong Taylor method (2) for the linear test equation (43) is MS-stable if $\bar{R}_{1}(p, q, z, c)<1$.

Applying the $\theta$-Taylor method (3) to the test equation (43), we obtain

$$
x_{n+1}=R_{2}\left(a, b, c, \Delta, \Delta W_{n}, \Delta N_{n}, \theta\right) x_{n},
$$

where

$$
\begin{aligned}
& R_{2}\left(a, b, c, \Delta, \Delta W_{n}, \Delta N_{n}, \theta\right) \\
& =\frac{1}{1-a \theta \Delta}\left[1+\left((1-\theta) a-\frac{1}{2} b^{2}\right) \Delta\right. \\
& +b \Delta W_{n}+\frac{1}{2} b^{2}\left(\Delta W_{n}\right)^{2}+\frac{1}{2}\left(2 c-c^{2}\right) \Delta N_{n} \\
& \left.+b c \Delta W_{n} \Delta N_{n}+\frac{1}{2} c^{2}\left(\Delta N_{n}\right)^{2}\right] .
\end{aligned}
$$

Then the MS-stability function of the $\theta$-Taylor method is

$$
\begin{aligned}
\bar{R}_{2}(p, q, z, c, \theta) & \\
=\frac{1}{(1-p \theta)^{2}} & {\left[\bar{R}_{1}(p, q, z, c)\right.} \\
& -2 p \theta+p^{2} \theta^{2}-2 p^{2} \theta \\
& \left.-2 p z \theta-p z^{2} \theta\right] .
\end{aligned}
$$


Thus, the $\theta$-Taylor method (3) for the linear test equation (43) is MS-stable if $\bar{R}_{2}(p, q, z, c)<1$.

Applying the balanced $\theta$-Taylor method (4) to the test equation (43), we obtain

$$
x_{n+1}=R_{3}\left(a, b, c, \Delta, \Delta W_{n}, \Delta N_{n}\right) x_{n},
$$

where

$$
\begin{aligned}
R_{3}\left(a, b, c, \Delta, \Delta W_{n}, \Delta N_{n}\right) & \\
=\left((1-a \theta \Delta) I+C_{n}\right)^{-1} & \\
\times[1 & +\left((1-\theta) a-\frac{1}{2} b^{2}\right) \Delta \\
& +b \Delta W_{n}+\frac{1}{2} b^{2}\left(\Delta W_{n}\right)^{2}+\frac{1}{2}\left(2 c-c^{2}\right) \Delta N_{n} \\
& \left.+b c \Delta W_{n} \Delta N_{n}+\frac{1}{2} c^{2}\left(\Delta N_{n}\right)^{2}+C_{n}\right] .
\end{aligned}
$$

Since $\mathbb{E}\left(R_{3}^{2}\right)$ is rather complex in the general case, we try to investigate the stability of balanced $\theta$-method (4) for the following two typical cases.

Case 1. Let $C_{0}=-a$ and $C_{1}=0$. Then, applying the balanced $\theta$-Taylor method (4) with $C_{n}=-a \Delta$ to the test equation (43), we obtain

$$
x_{n+1}=R_{3}^{\prime}\left(a, b, c, \Delta, \Delta W_{n}, \Delta N_{n}\right) x_{n},
$$

where

$$
\begin{aligned}
& R_{3}^{\prime}\left(a, b, c, \Delta, \Delta W_{n}, \Delta N_{n}\right) \\
& \begin{aligned}
=(1-a(1+\theta) \Delta)^{-1}[ & 1-a \theta \Delta-\frac{1}{2} b^{2} \Delta+b \Delta W_{n} \\
& +\frac{1}{2} b^{2}\left(\Delta W_{n}\right)^{2}+\frac{1}{2}\left(2 c-c^{2}\right) \Delta N_{n} \\
& \left.+b c \Delta W_{n} \Delta N_{n}+\frac{1}{2} c^{2}\left(\Delta N_{n}\right)^{2}\right] .
\end{aligned}
\end{aligned}
$$

Then the MS-stability function of the balanced $\theta$-Taylor method (4) with $C_{n}=-a \Delta$ is

$$
\begin{aligned}
& \bar{R}_{3}^{\prime}(p, q, z, c) \\
& =\frac{1}{(1-p(1+\theta))^{2}} \\
& \quad \times\left[1+q^{2}+\frac{1}{2} p q^{2}+\frac{1}{2} q^{4}-2 p \theta+p^{2} \theta^{2}\right. \\
& \quad+\left(2+c+c q^{2}+2 q^{2}-2 p \theta\right) z \\
& \quad+\left(2+2 c+\frac{1}{2} c^{2}+q^{2}-p \theta\right) z^{2} \\
& \left.\quad+(c+1) z^{3}+\frac{1}{4} z^{4}\right] .
\end{aligned}
$$

Thus, the balanced $\theta$-Taylor method (4) with $C_{n}=-a \Delta$ for the linear test equation (43) is MS-stable if $\bar{R}_{3}^{\prime}(p, q, z, c)<1$.

Case 2. Let $C_{0}=-a$ and $C_{1}=b^{2}$. Then, applying the balanced $\theta$-Taylor method (4) with $C_{n}=-a \Delta+b^{2}\left(\left(\Delta W_{n}\right)^{2}-\Delta\right)$ to the test equation (43), we have

$$
x_{n+1}=R_{3}^{\prime \prime}\left(p, q, c, J, \Delta N_{n}\right) x_{n},
$$

where

$$
\begin{aligned}
& R_{3}^{\prime \prime}\left(p, q, c, J, \Delta N_{n}\right) \\
& =\left(1-p \theta+q^{2}\left(J^{2}-1\right)\right)^{-1} \\
& \times\left[1-p \theta-\frac{3}{2} q^{2}+q J+\frac{3}{2} q^{2} J^{2}\right. \\
& \quad+\frac{1}{2}\left(2 c-c^{2}\right) \Delta N_{n}+q c J \Delta N_{n} \\
& \left.\quad+\frac{1}{2} c^{2}\left(\Delta N_{n}\right)^{2}\right]
\end{aligned}
$$

and $J$ is the standard Gaussian random variable $J=$ $\Delta W_{n} / \sqrt{\Delta} \sim N(0,1)$. Then the MS-stability function of the balanced $\theta$-Taylor method (5) with $C_{n}=-a \Delta+b^{2}\left(\left(\Delta W_{n}\right)^{2}-\Delta\right)$ is

$$
\begin{aligned}
& \bar{R}_{3}^{\prime \prime}\left(p, q, z, c, J, \Delta N_{n}\right) \\
& =\frac{1}{\sqrt{2 \pi}} \sum_{-\infty}^{+\infty} \frac{(\lambda \Delta)^{m} e^{-\lambda \Delta}}{m !} \\
& \quad \times \int_{-\infty}^{+\infty}\left(R_{3}^{\prime \prime}(p, q, c, x, m)\right)^{2} e^{-\left(x^{2} / 2\right)} \mathrm{d} x .
\end{aligned}
$$

Thus, the balanced $\theta$-Taylor method (4) with $C_{n}=-a \Delta+$ $b^{2}\left(\left(\Delta W_{n}\right)^{2}-\Delta\right)$ for the linear test equation (43) is MS-stable if $\bar{R}_{3}^{\prime \prime}(p, q, z, c)<1$.

For the case of $c=-1$ and $z=-1$, the MS-stable regions of the numerical methods for the test equation are plotted in Figures 1 and 2. Figure 1 shows the MS-stable regions of Taylor method, the $\theta$-Taylor method, and the balanced $\theta$ Taylor method with $C_{0}=-a$ and $C_{1}=0$ when $\theta=1 / 2$ and $\theta=1$. Figure 2 shows the MS-stable regions of Taylor method, the $\theta$-Taylor method, and the balanced $\theta$-Taylor method with $C_{0}=-a$ and $C_{1}=b^{2}$ when $\theta=1 / 4$ and $\theta=1 / 2$. It should be noted that the MS-stable regions are the areas below the plotted curves and symmetric about the $p$-axis. From Figures 1 and 2, it is observed that the MS-stable regions of the $\theta$ Taylor method and the balanced $\theta$-Taylor method increase as the parameter $\theta$ increases. The MS-stable properties of the $\theta$-Taylor method and the balanced $\theta$-Taylor method are better than the Taylor method. Furthermore, the MS-stable properties of the balanced $\theta$-Taylor method with $C_{0}=-a$ and $C_{1}=0$ are better than those of the $\theta$-Taylor method for all $\theta \in[0,1]$. In addition, the MS-stable properties of the balanced $\theta$-Taylor method with $C_{0}=-a$ and $C_{1}=b^{2}$ are better than those of the $\theta$-Taylor method when $\theta \leq 1 / 4$, and the MS-stable properties of the $\theta$-Taylor method are better than those of the balanced $\theta$-Taylor method with $C_{0}=-a$ and $C_{1}=b^{2}$ when $\theta \geq 1 / 2$. 
TABLE 1: Mean of the absolute errors for different values of $\Delta$ and different methods.

\begin{tabular}{lccccccccc}
\hline Methods & $\Delta$ & $2^{-8}$ & $2^{-7}$ & $2^{-6}$ & $2^{-5}$ & $2^{-4}$ & $2^{-3}$ & $2^{-2}$ \\
\hline \multirow{3}{*}{-Taylor } & $\theta=0$ & 0.0024 & 0.0048 & 0.0099 & 0.0186 & 0.0389 & 0.0911 & 0.6853 & 3.0473 \\
& $\theta=1 / 2$ & 0.0022 & 0.0043 & 0.0090 & 0.0177 & 0.0368 & 0.0805 & 0.2548 & 0.7407 \\
& $\theta=1$ & 0.0042 & 0.0083 & 0.0170 & 0.0342 & 0.0699 & 0.1454 & 0.3025 & 0.5568 \\
\hline Balanced & $\theta=0$ & 0.0021 & 0.0041 & 0.0083 & 0.0152 & 0.0317 & 0.0665 & 0.3852 & 1.5570 \\
$\theta$-Taylor & $\theta=1 / 2$ & 0.0076 & 0.0134 & 0.0223 & 0.0361 & 0.0619 & 0.1504 & 0.7024 & 3.1984 \\
& $\theta=1$ & 0.0075 & 0.0132 & 0.0217 & 0.0347 & 0.0582 & 0.1360 & 0.6285 & 2.6648 \\
\hline
\end{tabular}

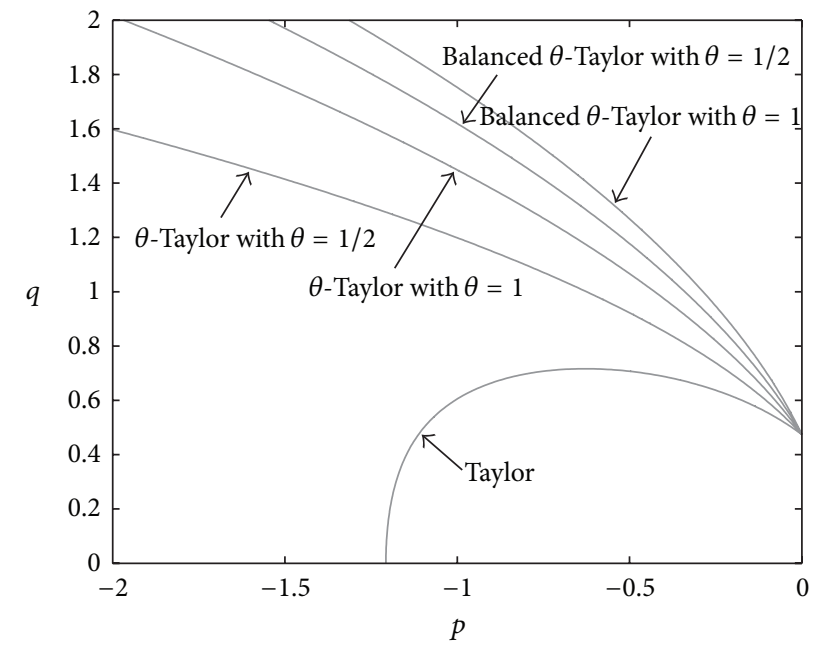

Figure 1: MS-stable regions of the $\theta$-Taylor methods and the balanced $\theta$-Taylor method with $C_{0}=-a$ and $C_{1}=0$.

\section{Numerical Examples}

In this section, we conduct some simulation to demonstrate the convergence of the proposed implicit Taylor numerical solutions (3) and (4) for the equation system (43) with the coefficients $a=-4, b=1, c=-0.5$ and the jump intensity $\lambda=2$. We compare the explicit solutions with the numerical approximations for the step-sizes $\Delta=2^{-1}, 2^{-2}, \ldots, 2^{-8}$. To measure the accuracy and convergence property of the proposed methods, we compute mean of the absolute errors as

$$
e=\frac{1}{2000} \sum_{i=1}^{2000}\left|x_{N}^{(i)}-x^{(i)}\left(t_{N}\right)\right|
$$

In Table 1, we report the simulated errors of the $\theta$-Taylor method and the balanced $\theta$-Taylor method with $C_{0}=1$ and $C_{1}=1$ for different values of $\theta$ and $\Delta$. Note that the Taylor method is a special case of the $\theta$-Taylor method with $\theta=0$. From Table 1 , we know that the accuracy of the $\theta$-Taylor method with $\theta=1 / 2$ and the balanced $\theta$-Taylor method with $\theta=0$ is higher than that of the Taylor method. The accuracy of the balanced $\theta$-Taylor method with $\theta=0$ is the highest for $\Delta \leq 2^{-2}$. When $\theta \geq 1 / 2$, the accuracy of the $\theta$-Taylor

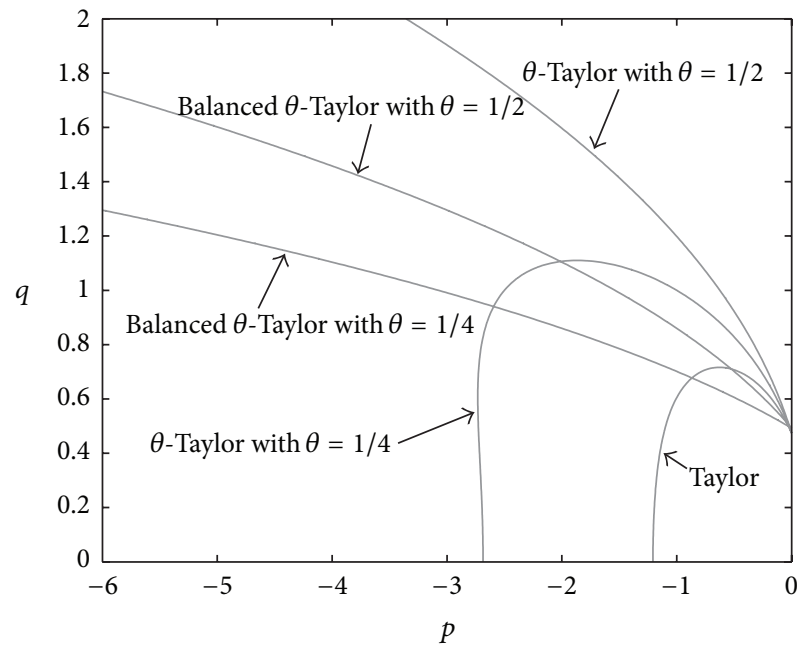

FIGURE 2: MS-stable regions of the $\theta$-Taylor methods and the balanced $\theta$-Taylor method with $C_{0}=-a$ and $C_{1}=b^{2}$.

method is higher than that of the balanced $\theta$-Taylor method with $C_{0}=1$ and $C_{1}=1$.

\section{Conclusions}

In this paper, we introduce two kinds of the implicit methods, the $\theta$-Taylor method and the balanced $\theta$-Taylor method, for solving stochastic differential equations with Poisson jumps. It is proved that the proposed numerical methods have a strong convergence order of 1.0. Moreover, the MS-stable regions of the proposed numerical methods are derived for a linear scalar test equation and it is demonstrated that the $\theta$-Taylor method and the balanced $\theta$-Taylor method have better stable properties than the Taylor method. As has been confirmed by the theoretical and the numerical results, the proposed numerical methods perform satisfactorily in solving SDEJs.

\section{Conflict of Interests}

The authors declare that they have no conflict of interests regarding to the publication of this paper. 


\section{References}

[1] D. J. Higham, "Mean-square and asymptotic stability of the stochastic theta method," SIAM Journal on Numerical Analysis, vol. 38, no. 3, pp. 753-769, 2000.

[2] D. J. Higham and P. E. Kloeden, "Convergence and stability of implicit methods for jump-diffusion systems," International Journal of Numerical Analysis and Modeling, vol. 3, no. 2, pp. 125-140, 2006.

[3] D. J. Higham and P. E. Kloeden, "Strong convergence rates for backward Euler on a class of nonlinear jump-diffusion problems," Journal of Computational and Applied Mathematics, vol. 205, no. 2, pp. 949-956, 2007.

[4] X. Mao, Y. Shen, and A. Gray, "Almost sure exponential stability of backward Euler-Maruyama discretizations for hybrid stochastic differential equations," Journal of Computational and Applied Mathematics, vol. 235, no. 5, pp. 1213-1226, 2011.

[5] L. Chen and F. Wu, "Almost sure decay stability of the backward Euler-Maruyama scheme for stochastic differential equations with unbounded delay," Applied Mechanics and Materials, vol. 235, pp. 39-44, 2012.

[6] L. Hu and S. Gan, "Mean-square convergence of drift-implicit one-step methods for neutral stochastic delay differential equations with jump diffusion," Discrete Dynamics in Nature and Society, vol. 2011, Article ID 917892, 22 pages, 2011.

[7] D. J. Higham and P. E. Kloeden, "Numerical methods for nonlinear stochastic differential equations with jumps," Numerische Mathematik, vol. 101, no. 1, pp. 101-119, 2005.

[8] X.-H. Ding, Q. Ma, and L. Zhang, "Convergence and stability of the split-step $\theta$-method for stochastic differential equations," Computers \& Mathematics with Applications, vol. 60, no. 5, pp. 1310-1321, 2010.

[9] X. Wang and S. Gan, "B-convergence of split-step one-leg theta methods for stochastic differential equations," Journal of Applied Mathematics and Computing, vol. 38, no. 1-2, pp. 489-503, 2012.

[10] E. Buckwar and T. Sickenberger, "A comparative linear meansquare stability analysis of Maruyama- and MILstein-type methods," Mathematics and Computers in Simulation, vol. 81, no. 6, pp. 1110-1127, 2011.

[11] G. N. Milstein, E. Platen, and H. Schurz, "Balanced implicit methods for stiff stochastic systems," SIAM Journal on Numerical Analysis, vol. 35, no. 3, pp. 1010-1019, 1998.

[12] K. Burrage and T. Tian, "The composite Euler method for stiff stochastic differential equations," Journal of Computational and Applied Mathematics, vol. 131, no. 1-2, pp. 407-426, 2001.

[13] T. H. Tian and K. Burrage, "Implicit Taylor methods for stiff stochastic differential equations," Applied Numerical Mathematics, vol. 38, no. 1-2, pp. 167-185, 2001.

[14] G. N. Milstein and M. V. Tretyakov, Stochastic Numerics for Mathematical Physics, Scientific Computation, Springer, Berlin, Germany, 2004.

[15] H. Schurz, "Convergence and stability of balanced implicit methods for systems of SDEs," International Journal of Numerical Analysis and Modeling, vol. 2, no. 2, pp. 197-220, 2005.

[16] C. Kahl and H. Schurz, "Balanced Milstein methods for ordinary SDEs," Monte Carlo Methods and Applications, vol. 12, no. 2, pp. 143-170, 2006.

[17] J. Alcock and K. Burrage, "A note on the balanced method," BIT: Numerical Mathematics, vol. 46, no. 4, pp. 689-710, 2006.

[18] S. S. Ahmad, N. Chandra Parida, and S. Raha, "The fully implicit stochastic- $\alpha$ method for stiff stochastic differential equations,"
Journal of Computational Physics, vol. 228, no. 22, pp. 82638282, 2009.

[19] M. A. Omar, A. Aboul-Hassan, and S. I. Rabia, "The composite Milstein methods for the numerical solution of Stratonovich stochastic differential equations," Applied Mathematics and Computation, vol. 215, no. 2, pp. 727-745, 2009.

[20] P. Wang and Z.-X. Liu, "Stabilized Milstein type methods for stiff stochastic systems," Journal of Numerical Mathematics and Stochastics, vol. 1, no. 1, pp. 33-44, 2009.

[21] P. Wang and Z. Liu, "Split-step backward balanced Milstein methods for stiff stochastic systems," Applied Numerical Mathematics, vol. 59, no. 6, pp. 1198-1213, 2009.

[22] L. Hu and S. Gan, "Convergence and stability of the balanced methods for stochastic differential equations with jumps," International Journal of Computer Mathematics, vol. 88, no. 10, pp. 2089-2108, 2011.

[23] A. Haghighi and S. M. Hosseini, "A class of split-step balanced methods for stiff stochastic differential equations," Numerical Algorithms, vol. 61, no. 1, pp. 141-162, 2012.

[24] X. Wang, S. Gan, and D. Wang, "A family of fully implicit Milstein methods for stiff stochastic differential equations with multiplicative noise," BIT: Numerical Mathematics, vol. 52, no. 3, pp. 741-772, 2012.

[25] L. Hu and S. Gan, "Numerical analysis of the balanced implicit methods for stochastic pantograph equations with jumps," Applied Mathematics and Computation, vol. 223, pp. 281-297, 2013.

[26] L. Hu, S. Gan, and X. Wang, "Asymptotic stability of balanced methods for stochastic jump-diffusion differential equations," Journal of Computational and Applied Mathematics, vol. 238, pp. 126-143, 2013.

[27] Y. Maghsoodi, "Mean square efficient numerical solution of jump-diffusion stochastic differential equations," Sankhya: The Indian Journal of Statistics, vol. 58, no. 1, pp. 25-47, 1996.

[28] Y. Maghsoodi and C. J. Harris, "In-probability approximation and simulation of nonlinear jump-diffusion stochastic differential equations," IMA Journal of Mathematical Control and Information, vol. 4, no. 1, pp. 65-92, 1987. 


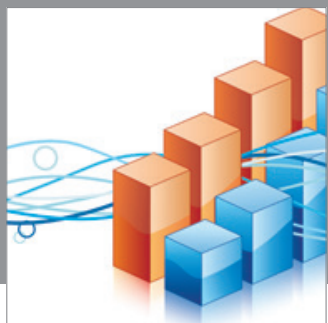

Advances in

Operations Research

mansans

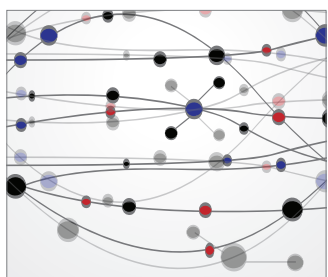

The Scientific World Journal
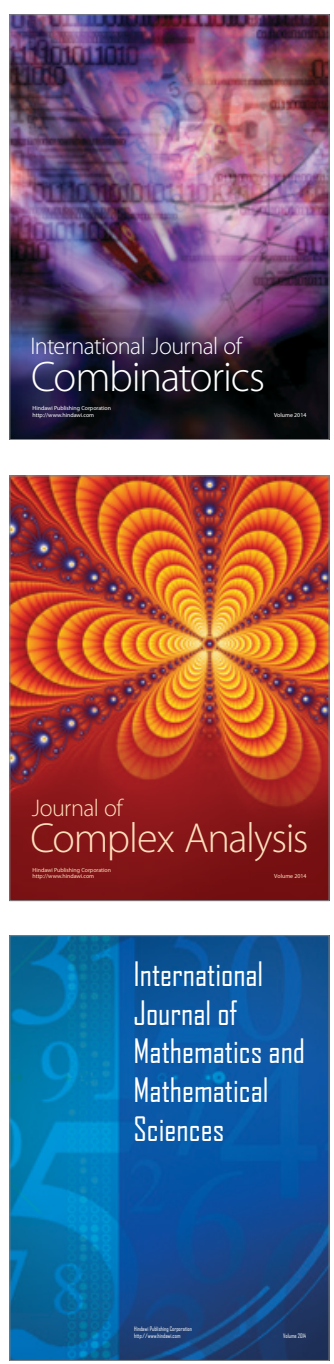
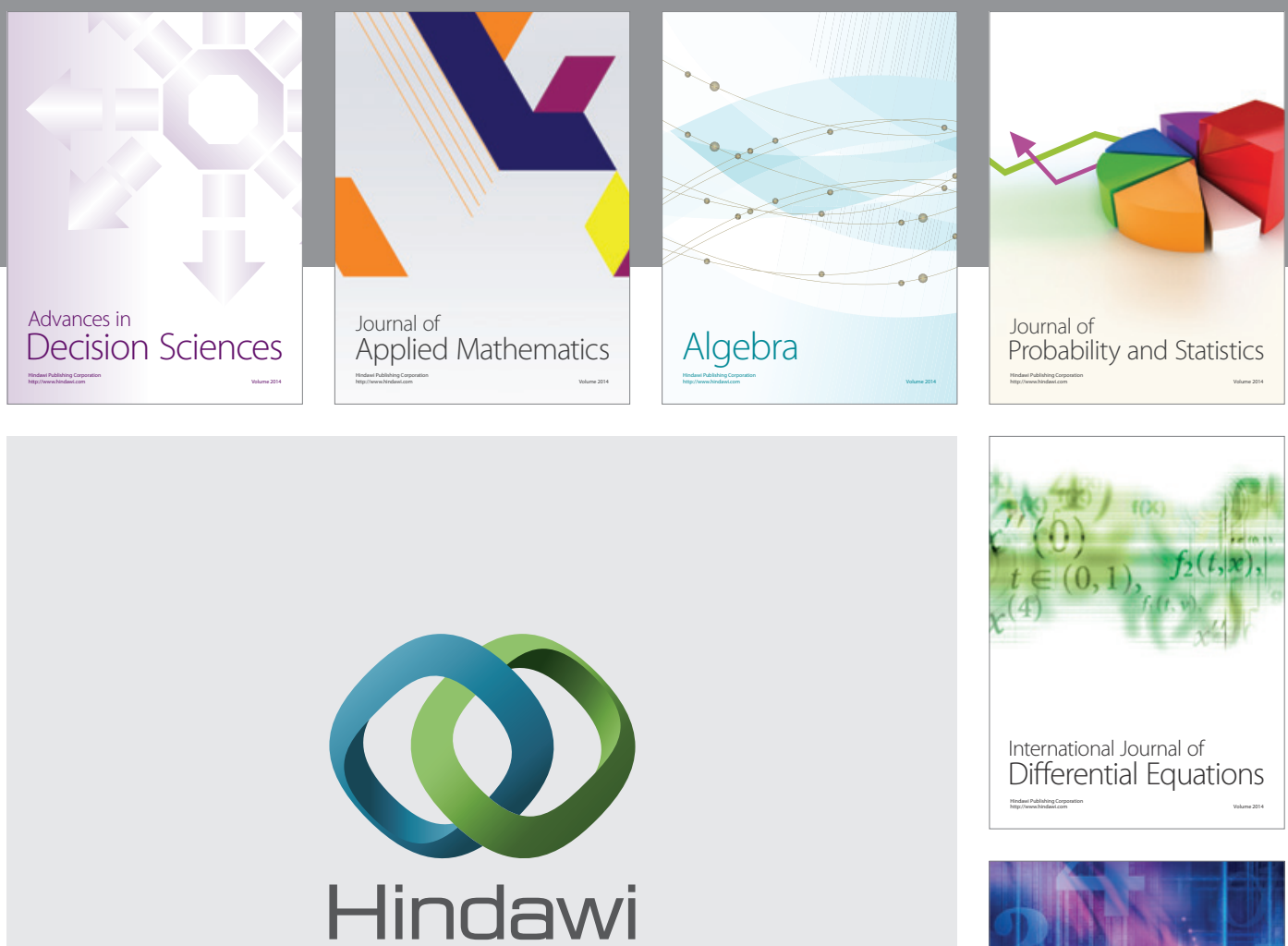

Submit your manuscripts at http://www.hindawi.com
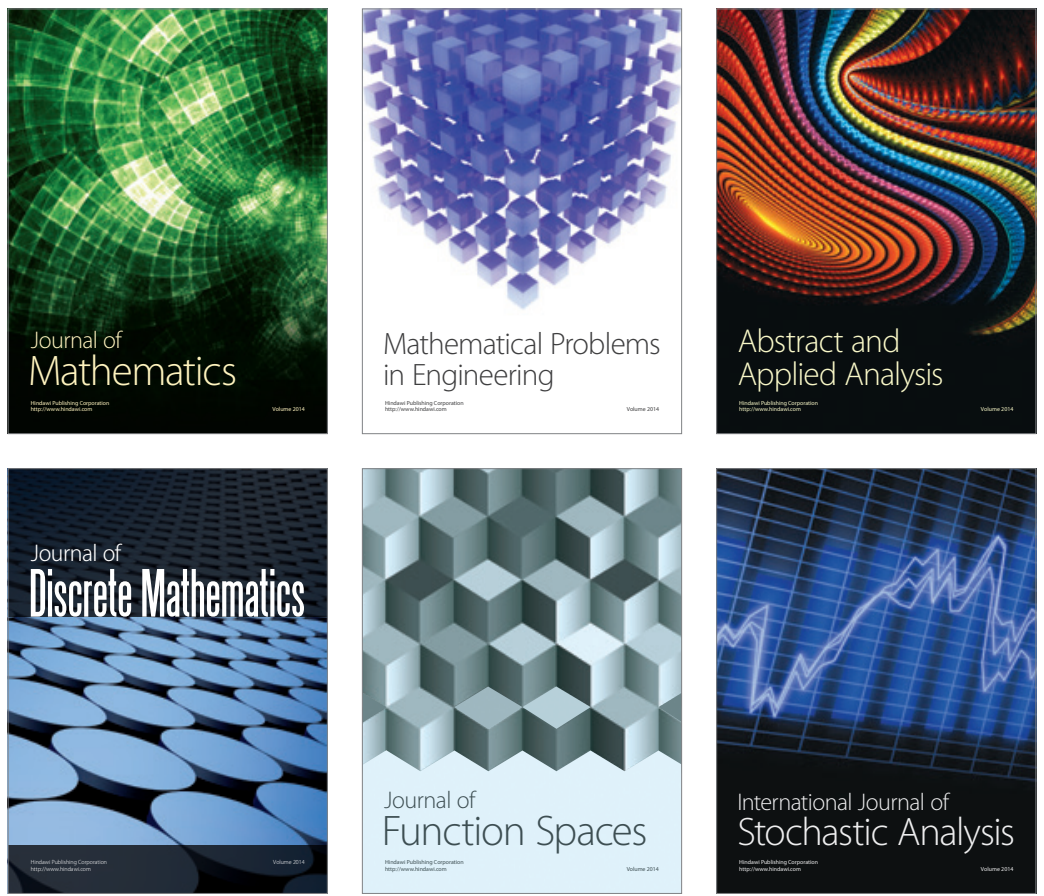

Journal of

Function Spaces

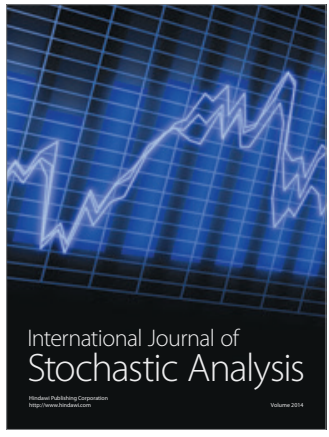

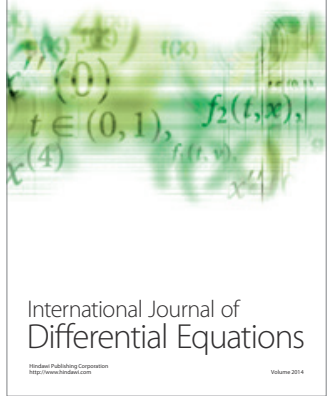
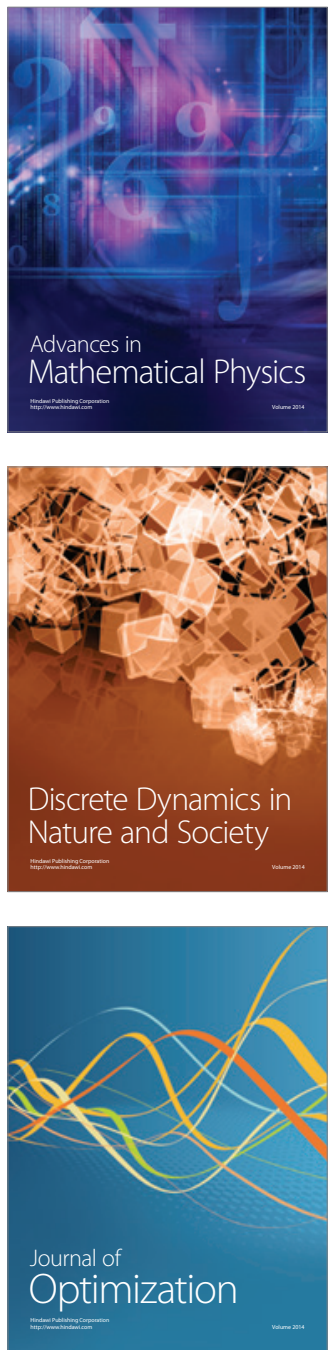